\title{
Stromab - Gedanken zur Hermeneutik biblischer Texte im Kontext der neueren angelsächsischen Diskussion
}

J A Loader

(Universität Wien und Unisa)

\begin{abstract}
Downstream - On Biblical hermeneutics in the context of the recent Anglo-Saxon debate

This paper, delivered at the 2000 meeting of the Rudolf Bultmann Society at Hofgeismar in Germany, offers a survey of recent developments in literary criticism in the English-speaking world. Contributions to the systematic reflection on issues relevant for theology are also considered and the author's own proposal is outlined. It is argued that the hermeneutic character of theology remains necessary in the Christian tradition. This is, however, not founded on the proposition of the linguistic nature of revelation, but on the fact that theology is the reflective human speaking about God.
\end{abstract}

\section{EINLEITUNG}

Die Hermeneutik wurde von ihrem Thron gestoßen, und wer heute noch nach ihr fragt, beweist damit nur, daß er von gestern ist.

Dieses aus dem Jahre 1971 rührende dictum von Klaus Scholder (1971:1) kann für die vorliegenden Ausführungen als programmatisch angesehen werden. Dazu sind aber drei Vorbemerkungen . notwendig.

\subsection{Vorbemerkung: Fragestellung und Ziel}

Obwohl die eben zitierte Behauptung in Ulrich Körtners Aufsatz Perspektiven hermeneutischer Theologie eher beiläufig zitiert wird, scheint mir die dadurch thematisierte Problematik für seine Darstellung zum Programm einer "Hermeneutischen Theologie" im Rahmen der Rudolf-BultmannGesellschaft doch grundlegend zu sein (Körtner 1999:263). An ihrer Wurzel steht nämlich die Frage nach der Berechtigung eines historischen bzw. formgeschichtlichen Zugangs zum biblischen Text. Es ist Aufgabe folgender Überlegungen, in diesem Zusammenhang vor dem Hintergnund der neueren angelsächsischen Debatte einen Beitrag zu liefern. Freilich, das Motto-Zitat ist negativ gemeint, nicht weil ich die Realităt dieser Palastrevolution bezweifle, sondern weil ich die vorausgesetzte Synonymität von "gestern" und "obsolet" zur Diskussion stellen möchte. Das wiederum in doppelter Hinsicht: Einmal bezüglich der Frage, ob Theologen/innen, die 
diese Frage "gestern" stellten, sich deshalb geirrt haben müssen. Zum anderen bezüglich der Frage, ob die Beschäftigung mit den biblischen Texten je von ihrem "Gestern" abgetrennt gedacht werden kann. Es gilt, den oft geleugneten aber immer wieder praktisch festgehaltenen Gegensatz zwischen den als "historisch" bzw. "nicht-historisch" gedachten Konzepten "diachron" und "synchron" in diesem Zusammenhang noch einmal zu problematisieren.

\subsection{Vorbemerkung: Begrenzung des Blickfeldes}

Solches vor dem Hintergrund der Tradition englischsprachige "literary criticism" biblischer Texte oder mit besonderem Hinweis auf sie zu tun, fordert aus mindestens drei Gründen einige Einschränkungen:

- Zunächst infolge des Charakters dieser Tradition, die nicht sosehr wegen ihrer umfassenden Werke theoretischer Art, sondern vielmehr wegen der Fülle ihrer praktischen Applikationen großen Einfluß hat. Daher stehen Studien dieser Art im Mittelpunkt der gebotenen Beschreibung. Andererseits müssen jedoch zwei theoretisch-argumentative Ansätze von James Barr und David Kelsey berücksichtigt werden, weil gerade sie für den ästhetischen Aspekt unseres Themas gravierende Impulse liefern.

- Zweitens ist damit zu rechnen, daß die zur Kennzeichnung der Richtungen, Modalitäten, Methoden und Techniken verwendete Terminologie sehr verwirrend wirkt, nicht zuletzt in der deutschsprachigen Literatur. Bald wird von "New Literary Criticism", bald von "New Criticism" als übergreifende Begriffe gesprochen. Oft ist aber nicht klar, ob beide nicht mit etwas zusammenfallen, das häufig "synchronic reading" genannt wird, ob sie mit dem "Bible-as-Literature-Approach" gleichbedeutend sind, ob es sich um eine Bewegung oder eine Methode handelt, und wie sich alles das zur "Rhetorical Criticism" oder zur "Narrative Criticism" verhält. Das erfordert ein gewisses $\mathrm{Ma}$ an Freiheit im deskriptiven ersten Teil meiner Darlegungen, damit wir nicht in den Bemühungen um eine möglichst genaue Abgrenzung von Schulen steckenbleiben.

- Schließlich muß angesichts dieser Sachlage wie auch der Fülle des Stoffes, auf eine Diskussion einiger nicht genuin angelsächsischer Ansätze verzichtet werden. So ist $z$.B. auf den sogenannten französischen Strukturalismus zu verweisen, der sich zwar unter biblischen Interpreten in den Vereinigten Staaten' und Britannien ${ }^{2}$ einen Platz eroberte, aber hinsichtlich der uns interessierenden Thematik keinen eigenständigen Beitrag

\footnotetext{
I Z.B. die Arbeit von Patte (1976), in der der Einfluß von A. Greimas auffallt, sowie seine Übersetzung von Jean Callound (1976), in der die gleiche Tradition vermittelt wird.

2 Eine gute Illustration, die auch weitere angelsächsische Vertreter vorführt, gibt Hawkes (1977).
} 
entwickelte, der über die typisch nordamerikanischen "approaches" hinausginge. Ebenso wird die typisch deutsche "Neue Hermeneutik", die mit ihrer Betonung des "Sprachgeschehens" auch in Amerika (Beispiele in Wellek \& Warren 1963) und Südafrika ${ }^{3}$ beeindruckte, nicht mehr berücksichtigt.

\subsection{Vorbemerkung: Definition}

In der Hermeneutik als Wissenschaft vom Verstehen geht es in ihrer biblischen Gestalt um das Verstehen von biblischen Texten. Dementsprechend wird sie entweder ganz eng als Regeln für die Exegese definiert oder ganz weit als ein Aspekt der literarischen Hermeneutik der allgemeinen Hermeneutik eingegliedert, in der über Verstehensbedingungen reflektiert wird, sodaß nur ihr Gegenstand spezifisch ist (Deist 1985:1ff.). Man begegnet auch der Sicht, jedes Bemühen um Interpretation von Texten aus der Perspektive des hermeneutischen Zirkels sei "Hermeneutik" oder "hermeneutische Literaturwissenschaft"4. Gemeint ist in diesem Zusammenhang nicht der Begriff "hermeneutische Theologie" in der Linie Gadamer-Ebeling, wo Sprache die "Vollzugsweise der hermeneutischen Erfahrung" ist," sondern das Adjektiv "hermeneutisch" hat den Sinn, die Theologie als schriftauslegende Theologie zu qualifizieren. Also geht es um das é $\rho \mu \epsilon \nu \in \mathcal{u}_{\epsilon} \iota \nu,{ }^{6}$ um das Vermitteln biblischer Inhalte und daher um die sich daraus ergebenden Implikationen für und Forderungen an die Theo-

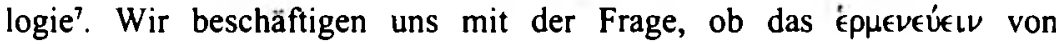
biblischen Texten angesichts der neueren angelsächsischen "Literary Criticism" noch möglich ist. Dazu ist nötig, daß wir uns eine kurze Übersicht über das Feld der neueren "Literary Criticism" verschaffen.

\section{NEUERE TENDENZEN DER “LITERARY CRITICISM" IM" ANGELSÄCHSISCHEN RAUM}

Wir überblicken die angedeuteten Tendenzen in drei grob übergreifenden Gruppen, die "New (Literary) Criticism", "Reader-Response Criticism" und Impulse aus der systematischen Reflexion dazu.

${ }^{3} \mathrm{Vgl}$. die positive Einschätzung von Groenewald (1969); vgl. auch Lategan (1970); Roberts (1973) (maßvoll kritisch).

${ }^{4}$ Bal, Luxemburg und Weststeijn (1983), wo das Verfahren von E Staiger als führendes Beispiel dieser allgemeinen Art des "hermeneutischen" Interpretierens angeführt wird.

$5 \quad$ Vgl. Gadamer (1960:445); Ebeling (1969); vgl. Körnter (1999:261); Oeming (1998:81-88).

${ }^{6} \mathrm{Vgl}$. den Beitrag vom zwar frankophonen, aber doch im angelsächsischen Raum tätigen J Grondin (1991:27f.), der auf die oft zitierte Stelle bei Platon (Ion. 534e) für einen antiken Beleg dieser Bedeutung verweist.

7 Von der Altemative "hermeneutische Theologie" oder "theologische Hermeneutik" wird unten noch die Rede sein. Vgl. Körtner (1999:263). 


\subsection{New Criticism}

Es gibt ebenso viel oder ebenso wenig eine einheitliche Größe "New Criticism", wie es eine einheitliche "historische Kritik" gibt. Dennoch sind bestimmte Eigentümlichkeiten, die diese von jener unterscheiden, klar zu erkennen.

Die Bezeichnung "New Criticism" stammt von E J Spingarn, der sie 1910 zum erstenmal verwendete, um damit eine textbezogene im Gegensatz zu einer textexternen Interpretation literarischer Produkte zu bezeichnen (Seymore-Smith 1988:579). Später, vor allem seit den dreißiger Jahren, hieß sie auch "New Literary Criticism", und sie versteht sich als Opposition gegen einen genetischen Vorgang, der ihres Erachtens nach dem fragt, was hinter dem Text liegt, statt nach Organisation, Stil und Statistik ("stylometric devices" und "word counting"). Um ihr Ziel zu erreichen, entwickelte die "New Criticism" eine Technik, das sogenannte "close reading", um eine Detailanalyse von allen linguistischen, stilistischen und strukturellen Aspekten des Textes zu gewinnen. Somit sind Einflüsse aus dem Strukturalismus und der Linguistik zu beobachten, so daß es m.E. nicht wünschenswert ist, allzu strenge Grenzlinien zwischen der strukturalistisch-linguistischen Kritik und der "New Criticism" zu ziehen". Diese Berührungen sind vor allem in der "Discourse Analysis" zu sehen, die den Text einer genauen Analyse seines Aufbaus unterzieht, und zwar mit Hilfe von linguistischen und strukturalistischen wie von stilistischen und statistischen Kriterien ${ }^{10}$. In der allgemeinen Literaturwissenschaft war das primär nicht anti-historisch gemeint, hatte aber von Natur aus diese Potenz, die v.a. auf dem Gebiet der biblischen Interpretation zu heftiger Kritik führte (Alter 1981:13; Oeming 1998:71). Das ist eine deutliche Analogie zur deutschen textimmanenten Interpretation liter-

\footnotetext{
${ }^{8}$ Eine gute lllustration bietet Ransom (1941), indem er eine heftige Auseinandersetzung mit Kollegen aus der Bewegung auslöste, weil die damals übliche "New Criticism" seiner Meinung nach nicht "ontologisch" genug wäre.

9 Damit ist nicht gesagt, daß beide Richtungen identisch sind. So ist die "New Criticism" von der Kunst um der Kunst willen begeistert, während der Strukturalismus dieses Konzept weitgehend unterminiert, indem er vom (bürgerlichen) konventionellen Charakter der Kunst überzeugt ist. Siehe J Barton(1984: 180-184) für eine vergleichende Diskussion.

${ }^{10}$ Z.B. jeweilige pronominale Korrespondenz, Wortgruppen und Satzbau; Ringkomposition und Wiederholungen; Stilfiguren wie Assonanz und Alliteration; Wortfrequenz und - distribution. Diese Arbeit wurde aus den USA, vor allem aus der BloomingtonSchule, angeregt und fand in den Bibelwissenschaften eine markante Ausprägung in der "diskursanalytischen Schule" der Universität von Pretoria, Südafrika, wo als fuhrende Figuren J P Louw (Neues Testament) und W S Prinsloo (Altes Testament) zu nennen sind. Vgl. Louw (1973:101-118); Prinsloo (1988; 1992:66-81). In Amerika selbst ist auf die einflußreiche Arbeit von R M Polzin und den Indianapolis-Kreis hinzuweisen. $\mathrm{Vgl}$. Polzin (1977) sowic die Arbeit des Kanadiers R C Culley (1974: 165-181; 1976).
} 
arischer Werke," weil sie sich mit der "surface structure" eines Textes und nur damit auseinandersetzt.

In diesem Kontext ist innerhalb der biblischen Wissenschaften eine amorphe Bewegung hervorgegangen, die mannigfache Perspektiven und Methoden anwendet, sich jedoch mit der Bibel literarisch als Literatur beschäftigt und daher "Bible-as-Literature-Movement" heißt. Ein Zentrum dieser Bewegung ist die britische Universität von Sheffield und das mit ihr assoziierte Journal for the Study of the Old Testament (Clines \& Exum 1993). Neuerdings verbinden sich hăufig in einer Person textzentrierte mit leserzentrierten Interessen, soda $\beta$ eine Trennung der "Bible-asLiterature-Movement" von anderen Ansätzen nicht (mehr) möglich ist" ${ }^{12}$.

Wir wenden uns nun drei spezifisch textorientierten Richtungen aus diesem Traditionsstrom zu.

\subsubsection{Rhetorical Criticism ${ }^{13}$}

"Rhetorics" meint in der angelsächsischen Welt nicht etwa in malam partem "die Kunst des Überredens"', sondern bezieht sich auf die dem Verstehen sprachlicher Äußerungen dienenden Mittel. Im Zentrum des Interesses steht also die Analyse jener sprachlichen Techniken, die in Texten angewandt werden, damit sie verstanden werden. Daher geht es um die Betonung des kommunikativen Aspekts von Texten in Anlehnung an die "speech act theory", wobei nicht von sprachlichen Techniken als grammatikalischen oder linguistischen Phänomenen die Rede ist, sondern im weiteren Sinne von der Verwendung der Sprache in zusammenhängenden literarischen Texten.

Aus der Tradition der nicht-genetischen Interpretation kommend, orientiert sich die "Rhetorical Criticism" am Text, zumal die in dem Text verwendeten Manöver analysiert werden. Aber da sie nicht an der Literatur" an sich interessiert ist, sondern vielmehr an der Literatur-in-Aktion, also an der Wirkung der Literatur, werden auch Autor und Empfänger ins Kalkül gezogen. Erforscht wird also die kommunikative Wirkung des Textes, wie er überzeugt bzw. versucht zu überzeugen. Das setzt aber im analytischen Verfahren eine soziale Perspektive voraus.

Wichtig ist die Erkenntnis, daß nicht nur sogenannte argumentative Gattungen zu "argumentieren" versuchen, sondern das auch in anderen

${ }^{11} \mathrm{Z}$ B Staiger (1955) mit seiner Hochschätzung von sprachlichen und Klangstrukturen; Ingarden (1972); Iser (1976); vgl. Schunack (1996:31).

12 Z.B. J C Exum, die viele Studien über die Bibel als Literatur schrieb und eine führende Figur in den Kreisen der feministischen Interpretation der Bibel ist. Vgl. Clines \& Exum (1993) und Exum (1996).

${ }^{13} \mathrm{Vgl}$. M Warner (1990); Mack (1990); Wuellner (1987:448-463).

14 Nach Schunack (1996:36) ist diese negative Konnotation schon in der "deutschsprachigen kulturellen und literarischen Offentlichkeit des 19. und 20. Jh." zu beobachten, wenn von "Rhetorik" die Rede ist. 
Textsorten, wie Erzählungen, der Fall ist. Indem die "Rhetorical Criticism" eine Sachebene von einer Aussageebene unterscheidet, wird es möglich, die eine als "story" und die andere als "discourse" zu analysieren. Nicht was erzählt wird (Inhalt), sondern wie das gemacht wird (argumentatives Verfahren), ist der Fokus. Dazu kommen literarische Fragen ins Spiel, wie "point of view", Stil, "setting" und die Auswahl aus dem zur Verfügung stehenden Arsenal von Motiven, Begriffen und Worten.

Seit der Initiative von James Muilenburg (1969:1-18) erfreut sich diese Lesestrategie einer breiten Anerkennung und wird auf dem Gebiet des Alten wie Neuen Testaments praktiziert's. Hier wurde die Einsicht aufgearbeitet, daß die Rhetorik in religiösen Texten zwar als Versuch zu überzeugen fungiert, aber nicht zwecks rationaler Beweisführung, sondern zur Verkündigung (so Warner 1990:136). So kann man die Evangelien als "stories" verstehen, die mittels ihrer rhetorischen Ebene einen kräftigen Diskurs mit dem Ziel zu überzeugen aufbauen. Dieses ist wiederum nichts anderes als das Kerygma der "story", das - anders als bei Bultmann innerhalb des Textes und nicht in einer vorangegangenen Erfahrung liegt (so z.B. Poland 1985:11ff.). Zudem wird damit dem religiösen Charakter der biblischen Texte ein fester Platz eingerăumt, was in den historischkritischen und anderen literaturwissenschaftlichen Interpretationsarten nicht der Fall sein muß.

Hierin besteht allerdings eine nicht unwichtige Unklarheit. Die "Rhetorical Criticism" will in der Tradition der "New Criticism" am Text orientiert sein, aber auch das, was bei Bultmann "Kerygma" heißt, bloßlegen und vermitteln. Nachdem sie als narrative Kritik die Textwelt analysiert hat, will sie offenbar auch die Textintention vermitteln und ist also in dieser Hinsicht hermeneutisch. Sie vermittelt angeblich den Sinn des Textes, wobei angesichts der großen Bedeutung des sozialen Aspekts von Sprache - ohne den die rhetorischen Konventionen und ihre Wirkungskraft nicht zu erkennen sind - uneinsichtig ist, worin der Unterschied zur Autorenintention liegt. Wo von einem Kommunikations-modell ausgegangen wird, ist also die Aufeinanderbezogenheit von Autor, Text und Leser gegeben. Deshalb steht die "Rhetorical Criticism" grundsätzlich der "Reader-Response Criticism" (s.u.) offen gegenüber.

\subsubsection{Narrative Criticism ${ }^{16}$}

Dieser Zugang zum Text wird m.E. von Schunack zurecht "in gewissem Sinne eine interpretative Konkretion der Rhetorik", und zwar von "poetischen" Texten, genannt. Zeigt sich die nahe Verwandtschaft zur "Rhetorical Criticism" bereits dadurch, daß Oeming beide als austauschbare Begriffe (sogar synonym zur "New Criticism" und "Synchronic

${ }^{15} \mathrm{Z}$ B Jackson \& Keßler (1974); weitere Literatur bei Schunack (1996:28.36-42).

${ }^{16} \mathrm{Vgl}$ Powell (1990); Alter (1981). 
Approach") verwendet (Oeming 1998:70), tritt sie v.a. im vielfachen Gebrauch von gleichen literarischen Phänomenen, wie "point of view", "setting" und rhetorischen Strategien klar hervor.

Die entscheidende Basis dieser Kritik ist das Konzept der "erzählten Welt" des Textes, die eine geschlossene Einheit bildet und nur in sich referiert. Daher ist man nicht am wirklichen Autor und dessen Intention interessiert, wohl aber am Erzähler, der ein rhetorisches Element darstellt, mit dem die Erzählung den Betreffenden (ebenfalls ein rhetorisches Konstrukt) $)^{17}$ anspricht.

An der "Oberfläche" arbeitet die narrative Analyse mit etlichen Elementen, die ebenso in anderen textorientierten Methoden, wie im "close reading", in der Aufdeckung von Strukturmustem und Stilfiguren sowie Symbolen verwendet werden ${ }^{18}$. Die "story" wird in einem "plot" (Erzählzusammenhang) organisiert und, damit verbunden, werden die erzählerischen Personen charakterisiert. Hier tritt wieder ein rhetorisches Moment in Aktion, und zwar so, daß der Leser beeinflußt wird, sich mit den "good guys" zu identifizieren und gegenüber den "bad guys" Antipathie zu empfinden. Damit "verstrickt" (Schunack 1996:47) ${ }^{19}$ sich der Leser in die Erzählung, sein "Überzeugtwerden" wird angestrebt, und ein hermeneutischer Aspekt ist vorhanden.

Am deutlichsten zeigt sich die Beziehung zur "Rhetorical Criticism" darin, daß auch hier der "point of view", die Perspektive, von der aus sich die rhetorische Wirkung der Erzählung entfaltet, eine bestimmende Rolle spielt. Der ganze Text wird von hier aus so organisiert, daß an den Leser der Appell ergeht, sich die Intention des Textes zueigen zu machen. Es soll eine Botschaft vermittelt werden. Das läuft parallel zum Überzeugungscharakter des Textes, woran der "Rhetorical Criticism" so viel liegt, bedeutet aber wiederum, da $\mathrm{B}$ - bei aller Betonung ihrer textimmanenten Arbeitsweise - auch die narrative Analyse prinzipiell für eine Weiterführung offen ist, in der das hermeneutische Element eine wichtige Rolle spielt.

17 Jeweils noch vom impliziten Autor bzw. impliziten Leser zu unterscheiden, die vom Leser bzw. vom Text vorausgesetzte, auf das Verstehen des Textes ausgerichtete literarische Konstrukte sind.

${ }_{18}$ Z.B. Symmetrische Muster (wie ABCDdcba), Ringkomposition, der "pivot point" (Fokuspunkt), Parallelie, Kontrastierung, literarische Antizipation, Chiasmen usw.

${ }_{19}$ M.E. gilı dasselbe auch für nichterzählerische, poetische Texte, wo der Leser in den Text hineingezogen, also ästhetisch überwältigt wird und deshalb in eine hermeneutische Erfahrung aufgenommen wird. 


\subsubsection{Canon Criticism ${ }^{20}$}

Ein besonderer Fall der literarischen Kritik liegt im ungeheuer einflußreichen "Canonical Approach" vor. Normalerweise wird von dieser Bewegung als einer Einheit gesprochen, aber m.E. werden damit gewichtige Perspektiven übergangen oder unterbewertet. Von den Vertretern dieses Ansatzes könnte man zwar behaupten, alle zeigten gemeinsam, daß

"the canonical approach clearly belongs more to the realm of what in ordinary usage would nowadays be called ,literary" criticism than to that of the ,historical" study of texts, in that it is concerned with what the text means rather than with what it meant (Barton 1984:90)".

Ebenso könnte man von seiner Zugangsweise als solcher sagen, daß die "philosophischen Hintergründe ... hier ganz ähnlich wie die beim New Literary Criticism" (Oeming 1998:75) seien.

Dem ist aber nur mit Einschränkung zuzustimmen. Auf der einen Seite ist es richtig, daß die typische Betonung der Endgestalt des Textes und die damit verbundene Hochschätzung von größeren Zusammenhängen sowie literarischen Techniken und Kompositionstechniken die "Canon Critics" fest in die Tradition der "New Criticism" einbindet ${ }^{21}$. Auf der anderen Seite gibt es aber auch einen auffallenden Unterschied zwischen zwei Hauptströmungen innerhalb dieser Richtung, nämlich der Linie von Childs und der von Sanders.

Childs Anliegen ist grundlegend theologisch, weil er sein Programm entschieden zu dem Zweck entwarf, den Bruch zwischen der Bibelwissenschaft und der biblischen Theologie, für den die historische Kritik verantwortlich sei, zu heilen. Daher ist dieses Vorhaben ein konfessionelles Unternehmen, bei Childs selbst sogar reformiertes, und nicht an erster Stelle ein literarisches". Mit Oeming kann man es als "amerikanischen Pragmatismus" (Oeming 1998:76) einschätzen, was aber Kritik an einer gewissen Oberflächlichkeit impliziert. Diese liegt darin, daß der behauptete Verzicht auf textexterne Kriterien zugunsten einer textimmanenten

${ }^{20} \mathrm{Vgl}$. Childs (1979); Barr (1983) (v.a. 130ff. für eine scharf kritische Reaktion zu Childs); Sanders (1984); Brett (1991).

${ }^{21}$ Childs selbst weist auf die Verwandtschaft seiner Methode mit der "newer criticism", "various forms of structural analysis, and of rhetorical criticism" (Childs 1979:74) hin.

${ }^{22}$ Childs selbst (1979:74) sagt im gleichen Zusammenhang, wo er auf die Ähnlichkeit zwischen seinem Anliegen und den "literary" Methoden hinweist, daß es sich von anderen unterscheidet, "by interpreting the biblical text in relation to a community of faith and practice for whom it served a particular theological role as possessing divine authority". Die Bedeutung des Textes für heute ("what it means") ist also völlig abhängig vom historischen Urteil über die Meinungen und Intentionen der außerhalb des Textes stehenden alten kirchlichen Gesellschaften. 
Interpretation nicht durchgeführt wird und auch nicht durchführbar ist. Denn die kanonische Interpretation ist nur ab dem Moment möglich, in dem es einen Kanon gibt (Barton 1984: a.a.O). Aber welcher Kanon es sein soll ${ }^{23}$ oder: was der Kanon ist oder wiederum: wie die zu interpretierende Endgestalt des Textes konstituiert wird, wird nicht textimmanent, sondern von außerhalb des Textes herangetragen. Die Sicht Childs', daß der Gebrauch des masoretischen Textes von der gemeinsamen Tradition des Christentums und des Judentums her richtig sei (Childs 1979:72-74.659671), darf naiv genannt werden, ${ }^{24}$ ist aber textextern und steht in Widerspruch zu seinem textimmanenten Bekenntnis.

Die zweite Modalität der kanonisch orientierten Richtung ist die von J A Sanders, für den der Kanon nicht sosehr ein Text, sondern ein Prozeß ist. Auch wenn man aus dieser Perspektive mit "literarischen" Momenten, wie Kompositionsstruktur und Stil, arbeitet (wie dies ja immer auch von der historischen Kritik getan wurde), ist man am Werdegang des Textes interessiert. Wenn Sanders also "die Transformationsprozesse innerhalb des kanonischen Prozesses" und "den Verlauf der ständigen Adaptierung der Glaubenstradition an das jeweilige Leben" (Oeming 1998:77) studieren will, ist das ein historisches Interesse. Daher ist die Kombination dieses Ansatzes mit der Redaktionskritik, wie das z.B. in der Einleitung zum Alten Testament von Erich Zenger und seinen Mitarbeitern zum Ausdruck kommt, ${ }^{25}$ völlig verständlich. Die kanonische Sicht von Rolf Rendtorff illustriert, daß es in dieser kompositionsbewußten kanonischen Kritik - bei aller Hochschätzung des "Endtextes", der Komposition und des literarischen Zusammenhangs - noch immer um einen Aspekt oder eine Sonderform der historischen Kritik geht (Rendtorff 1992).

${ }^{23}$ Dieser m.E. sehr wesentliche Aspekt der Problematik wird in seiner Evaluation von Oeming eher beiläufig erwähnt.

${ }^{24}$ Zur Kritik vgl. Bart (1980:22). Zur Diskrepanz in Childs' Berufung auf Karl Barth s. auch Barr (1983:140-142). Barth arbeite nicht "from the context of the Christian canon", sondem nach ihm liege die Bedeutung des Textes "in the extrinsic realities, the theological real world, to which this pointed". Demgegenüber ist der Hinweis Bartons auf das Prinzip "was Christum treibet" als Luthers Kriterium für die Bestimmung des kanonischen Status aufschlußreich: Dieses Prinzip will vollig "internal biblical" sein (also "textimmanent", wie es Childs auffaßt), setzt aber voraus, daß einzelne Teile der Bibel - das jeweilige "was" - ohne Verweis auf die weiteren Zusammenhänge gelesen werden können, um zunăchst über ihre Kanonizität zu urteilen. Kurz: Wer kanonisch und textimmanent arbeiten will, muß scheitem; wer textimmanent arbeiten will, kann die kanonischen Zusammenhänge nicht so verabsolutieren, wie es Childs tut. Vgl. Sundberg (1964), für den die christliche Kirche bis zur Reformation keinen Kanon des Alten Testaments außer den griechischen anerkannt habe, und Loader (1979:10f.) über diese Problematik für konservative Theologie.

${ }^{25}$ Zenger (1996); weitere Beispiele aus dem deutschen Sprachbereich bei Oeming (1998:78). 
Immerhin ist die Einflußnahme der deutschen Redaktionskritik auf die sich in der Tradition des "New Criticism" zu Hause fühlende angelsächsische Kanonkritik und deren Rückwirkung auf die deutsche Sicht der Redaktionskritik ein Indiz dafür, daß die "Canon Criticism" in hermeneutischer Hinsicht nicht ganz problemlos ist. Die Bibel soll als Kanon interpretiert werden, damit sie verstanden werden kann. Dieses Verstehen aber ist ein theologisches Verstehen, das für Childs ein Versuch ist,

to heal the breach between biblical criticism and theology, and it assumes (at least for the purpose of method) that the interpreter is not a detached, neutral critic, free from religious commitment, but a believer, trying to apply the biblical text to the contemporary life of the Church (Barton 1984: 90).

Daher treffen die wissenschaftlichen Vorteile der kanonischen Auslegung, wie sie Oeming beschreibt, durchaus auf den redaktionskritischen Aspekt der historischen Kritik zu, während die unerfreuliche Frontstellung gegen die historische Kritik nicht ihr einziges Defizit ist. Gerade der hermeneutische Bezug der "Canon Criticism" ist das Interessante, weil hierin eine Inkonsequenz liegt: Die Bibel soll nicht nur in der Vergangenheit etwas bedeutet haben, sondern auch jetzt etwas bedeuten. Bedeutung soll vermittelt werden, aber der zu vermittelnde Sinn wird nicht aus dem Text selbst geholt, sondern aus einer Reihe von Intentionen, die in der Geschichte hinter der Formation der Kanons standen.

\subsection{Reader-response criticism (vgl. detweiler 1985)}

Zurecht betrachtet Schunack die "Reader-Response Criticism" eher als eine breite Sammlung von Textzugängen denn als eine Methode:

"So können unter dem Titel "Reader-Response Criticism" sehr unterschiedliche Interpretationsverfahren zusammengefaßt oder als dessen Ausprägungen aufgefaßt werden: Rhetorisch-kommunikative, semiotische und strukturalistische, phănomenologische, subjektive und psychoanalytische, soziologische und historische sowie hermeneutische ${ }^{26 "}$.

\footnotetext{
${ }^{26}$ Schunack (1996:49), unter Berufung auf Suleiman \& Crosman (1980:6ff). Ähnlich scheint das Urteil von Oeming, dessen Behandlung der "an den Lesern orientierte[n] Methoden" sechs verschiedene Lesestrategien umfaßt" (Oeming 1998:89-139 mit Verweis auf Dohmen \& Stemberger (1996: 193), wo es heißt, "Sinn und Bedeutung werden vom jeweiligen Leser und Interpreten konstruiert").
} 
Dieses Arsenal von Methoden ist eine Weiterführung der Einsichten, die aus der "Narrative Criticism" bzw. aus der als "literary" gedachten Kritik gewonnen wurden. Der Fokus liegt beim Leser bzw. beim Lesen, aber wie der Name schon andeutet, wird dieses dynamisch als Prozeß gesehen.

Auch hier wird grundsätzlich mit einem Kommunikationsmodell gearbeitet, also mit der Interaktion von Text und Leser. Dies kann auch mit Rücksichtnahme auf die in der "Narrative Criticism" wichtigen Unterscheidungen zwischen realen und impliziten Autoren und Lesern geschehen, während der Unterschied zwischen "story" und "discourse" bzw. Argumentation sowie das minutiöse "close reading" häufig herangezogen werden.

Verstehen ereignet sich aber in der Wechselbeziehung von Text und Leser, indem der Leser durch die Textorganisation zu einer Anteilnahme am Text veranlaßt ("involved") wird (Moore 1989:63). Somit ist die Affinität der "Reader-Response Criticism" mit der rezeptionsästhetischen Dynamik von Wolfgang Iser deutlich (Iser 1984:50; vgl. Körtner 1994:133). Der Südafrikaner Bernhard Lategan betont in dieser Dynamik ganz stark die Wechselseitigkeit von Text und Leser: Der Leser tritt an den Text mit bestimmten Vorverständnissen, Erwartungen und Wünschen heran, die Grundlegendes zur Bedeutung beitragen, die der Text in dieser "response" gewinnt. Aber der Leser kann auch nicht machen, was er will, weil Direktiven im Text kodiert sind, die seine Aktualisierung bestimmen ${ }^{27}$. In dieser Einsicht liegt m.E. die Relevanz der "Reader-Response Criticism" für die Fragestellung, die uns nun beschäftigt. Angesichts der Betonung des Beitrags, den nach diesem Ansatz der Leser leistet, wird die hermeneutische Frage in der Auseinandersetzung mit der "Reader-Response Criticism" interessant. Wenn mit der zentralen Stellung dieses Beitrags gemeint ist, daß die ästhetische Erfahrung des Lesers zur Erfassung des Textsinnes. ausreicht, wird damit die Ansicht vertreten, die Hermeneutik sei obsolet. Denn in diesem Fall gäbe es keine ursprüngliche Intention mehr, die vermittelt werden soll.

Diese Sicht wird eindrucksvoll in einer letzten Radikalisierung der leserorientierten Textauffassungen vertreten, auf die hier wegen ihrer eigenen Leserbezogenheit sowie ihrer negativen Beantwortung der eben erwähnten "hermeneutischen Frage" noch hingewiesen werden soll. Es handelt sich um die Dekonstruktion. Der Leser gilt schlechthin als Subjekt aller erfahrbaren Bedeutung bzw. alles erfahrbaren Sinns. Hiervon geben Ulrich Körtner und Gerd Schunack Beschreibungen (Körtner unter Verweis auf die "exemplarische" Debatte zwischen H-G Gadamer und Jacques Derrida ${ }^{28}$. In dieser Hinsicht erscheint Derrida angesichts der vielfältigen intertextuellen Bezüge eines Textes als "Antihermeneut"

${ }^{27}$ Lategan (1989:3ff). Siehe Anm. 3 oben zu seiner früheren Schätzung der "Neuen Hermeneutik".

${ }^{28}$ Körtner (1999:262-264); vgl. Forget (1984); Schunack (1996:52-55). 
(Oeming 1998:90), aber ähnliches läßt sich auch von der Dekonstruktion biblischer Texte sagen ${ }^{29}$.

Ich möchte unten in meinen eigenen Überlegungen noch Gründe anführen, warum ein solches "für-obsolet-Halten" in innere Probleme verstrickt wird, wenn der geschichtliche und soziale Kontext der Interpretation in Betracht gezogen wird. Aber bereits unter Einbeziehung der rezeptionsästhetischen Dialektik von Text und Leser ist festzustellen, daß sich der hermeneutische Umgang mit dem biblischen Text nicht so leicht beseitigen läßt.

Zwar ist das responsum des Lesers, also seine Reaktion auf den Text, ausschlaggebend für die Bedeutung, die der Text in dieser Begegnung erhält, aber trotzdem soll der Text seine Bedeutung nicht einfach vom Leser empfangen, sondern auch eine Bedeutung für den Leser gewinnen. Das ist aber das hermeneutische Verfahren die hermeneutische Erfahrung. Bedeutung soll dem Leser vermittelt werden Deshalb ist es ohne weiteres klar, da $\beta$ bedeutende Ansätze der Hermeneutik (allerdings nicht nur der neutestamentlichen Hermeneutik) in der "Reader-Response Criticism" vorgegeben sind (Schunack 1996:51).

\subsection{Impulse aus der systematischen Reflexion}

Wir wenden uns nunmehr zwei systematischen Beiträgen aus der angelsächsischen Welt zu, die mir besonders aufschlußreich für uns Thema scheinen, zunächst den Überlegungen von David $\mathrm{H}$. Kelsey aus Yale und dann dem immens einflußreichen Werk des britisch-amerikanischen Theologen und Kritikers James Barr.

\subsubsection{Die Bibel und heutiger Sinn}

David Kelsey veröffentlichte in der Mitte der siebziger Jahre eine Studie, die bald hochgeschätzt wurde und sich einer breiten Wirkung erfreuen konnte. ${ }^{30}$ Die Frage nach der Autorität der Bibel ist hier eine Frage nach der Relation zwischen einer theologischen Position, die sich auf die Bibel bezieht, und der Bibel selbst. “... what does it mean when people say that a theological position is, or is not, in accord with scripture?" (Barr 1979:39) Also: Wie verhält sich die Bedeutung eines theologischen Konstrukts, das sich irgendwie auf die Bibel beruft zur Bedeutung der Bibel selbst?

Diese Frage wird unter verschiedenen Gesichtspunkten behandelt, von denen folgende für uns relevant sind:

${ }^{29} \mathrm{Vgl}$. Nethöfel (1992) und die Kritik an ihm von Körtner (1999: 263). Neben den oft zitierten Werken von Culler (1982) und Jobling \& Moore (1992) sei des weiteren als Beispiele aus der Literaturflut nur auf zwei beachtenswerte südafrikanische Beiträge zum Thema verwiesen, nämlich Hunter (1991:362-372) und De Villiers (1991:145156).

${ }^{30}$ Kelsey (1975). Vgl. das Urteil Barrs (1979:39): “This is one of the most important contributions to the question of biblical authority made in the last decades". 


\section{"Construing the text"}

Zunächst zeigt Kelsey, daß ein theologisches Berufen auf die Schrift ${ }^{31}$ bzw. ein Versuch, die Schrift theologisch sinnvoll reden zu lassen, seinem Wesen nach nicht ein Berufen auf die Schrift selbst ist bzw. diese selbst nicht als ganze zum Reden bringt. Wenn Theologen sich auf die Schrift berufen, konstruieren sie vielmehr den zu verwendenden Text. Dieses wird anhand des Bibelgebrauchs sieben prominenter Vertreter von Theologien verschiedener Schattierungen aus dem theologischen Spektrums gezeigt. Das, was für den autoritativen Inhalt der Bibel gehalten wird, also das, was heute theologisch sinnvoll zu Menschen sprechen kann, darf nicht mit der Bibel selbst identifiziert werden. Es ist vielmehr ein vom Theologen in der Bibel wahrgenommenes Muster, also eine Konstellation, die nach Empfinden des Theologen den appellativen bzw. sinnvollen Aspekt der Schrift ausmacht.

Nach Benjamin B Warfield (1948) ist die Bibel nicht nur die Heilige Schrift, sondern auch dem Wort Gottes gleichzusetzen:

"Saving faith necessarily includes belief that certain doctrines are true. Since it is important that the belief be utterly confident, the truths must be utterly trustworthy. That creates the need for an utterly trustworthy authority determining what those doctrines are".

Daher schließt Kelsey (1975:21): "What is the authoritative aspect of scripture? The doctrines it teaches". Bevor Warfield sich auf den Text beruft, hat er einen "Text" konstruiert - die Dogmen, die nach seiner konservativ-protestantischen Überzeugung die Schrift liefert.

- So verfährt Kelsey auch mit Vertretern der "Biblical Theology Movement", die seit den dreißiger Jahren bis nach dem Zweiten Weltkrieg vor allem in Großbritannien und in den USA eine Menge von Theologumena auf angeblich biblische Begriffe gründete (Kelsey 1975:24ff.). Obwohl sein Hauptbeispiel $\mathrm{H}-\mathrm{W}$ Bartsch ist, führt er viele Beispiele aus der anglo-amerikanischen Szene an ${ }^{\mathbf{2}}$. Eine besondere Manifestation dieser Bewegung stellt die Arbeit von G E Wright (1952) dar (Kelsey 1975:3338). Sein Berufungsmuster in der Schrift ist deren Erzählcharakter, der aber nichts mit der narrativen Interpretationsweise zu tun hat, sondern als "confessional recital" der Schrift konstruiert wird. Was die Bibel zu

31 Kelsey spricht von der "Schrift" ("scripture"), um damit ihren funktionellen Charakter als heilige Schrift des Christentums, also als autoritatives Modell dieser Tradition, anzudeuten.

32 G A F Knight, J Marsh, J K S Reid, A Richardson, T F Torrance u.a.; Kelsey (1975:31). 
vermitteln hat, ist die Offenbarung Gottes als "inference of what actually happened in history". Die sinnvollen Aussagen der biblischen Begriffe sollen in diesem Rahmen evident gemacht werden (Wright 1952:44.57).

- Das sinnvolle Element, das theologisch zu kommunizieren ist, können bei Karl Barth (Kelsey 1975:39-50) verschiedene Dinge sein, aber nach Kelsey besteht eines der wichtigsten darin, daß Barth die biblischen Erzählungen als "identity descriptions" konstruiert. D.h., die Schrift liefert einen "agent", der für die Leser lebendig wird. Der Agent, die Person, mit der der Leser konfrontiert wird, Jesus, ist gemäß seinem Auftreten in der Geschichte zu verstehen und bringt ein "presence" mit sich, das uns "our normative link with God's self-disclosure" bietet.

- Nachdem er gezeigt hat, daß der "konstruierte Text" bei L S Thornton die "biblical images" sind, die dem modernen Geist adäquat sein können (Kelsey 1975:57-64), kommt Kelsey zu Paul Tillich, nach dem " $\mathrm{t}$ ] he subject matter of theology ... is the symbols given by the original revelatory experiences and by the tradition based on them" (Tillich 1963 Bd. 3:201). Diese Symbole haben einmal eine expressive Funktion, indem sie der ursprünglichen Offenbarung Ausdruck verleihen, aber ebenso eine "occasioning function", indem sie in Zusammenhang mit den existentialen Fragen, die der Mensch heute stellt, wieder neue - von den ursprünglichen abhängige - Symbole generieren können (Tillich 1963 Bd. 1:6-8; Kelsey 1975:71). Dieses Netzwerk von Symbolen ist also Tillichs Schrift, die besonders klar ursprünglichen Sinn in der jeweils neuen Situation vermittelt.

- Zu seiner letzten "case study" gibt Kelsey folgenden deutlichen Kommentar:

"Although it may be the only thing he has in common with them, Rudolf Bultmann shares with Thornton and Tillich the view that what is authoritative in the Bible are passages that express the revelatory and saving ,Christ event and occasion contemporary saving and revealing events (Kelsey 1975:74; Hervorhebung von mir)".

Er belegt aus der englischen Übersetzung Bultmanns Theologie des Neuen Testaments, daß das Anliegen der Theologie die Explikation des dem Glauben innewohnenden Selbstverständnisses ist. Er fährt fort:

"The paradigmatic expressions of Christian self-understanding are found in the New Testament. Bultmann appears to take them as normative because they are the earliest extant expressions of faith. Consequently, the theologian must turn to scripture to authorize his particular ways of explicating faith's self-understanding (Kelsey 1975:75)". 
Indem Bultmann die neutestamentlichen Expositionen der Versöhnung an diejenige der eschatologischen Existenz assimiliert, weil diese das Herz der Botschaft des Neuen Testaments ausmachen, kann er vorschlagen, die eschatologische Existenz zeige genau das, was nach Martin Heidegger authentische Existenz ist (Kelsey 1975:76f.). Die von Bultmann konstruierte Schrift bzw. das in ihr vorgefundene Muster, ist also die Fähigkeit, authentische Existenz anzubieten. Der vermittelnd-hermeneutische Charakter dieser Konstruktion ist wahrscheinlich am deutlichsten bei Bultmann zu sehen.

\subsubsection{Die ästhetischen und hermeneutischen Seiten der Schriftverwendung}

Die Entscheidung für einen die Bibel brauchbar und relevant machenden Aspekt ist nach Kelsey eine kreative Tat des imaginären Geistes, die das, worauf es ankommt, entscheidet, nämlich "the mode of God's presence pro nobis" (Kelsey 1975:163). Während dieses "imaginative judgment" freilich innerhalb eines bestimmten Kontextes (also in einer Tradition) gefällt wird, der schon von dieser Schrift mitgeformt wurde und seine Materialien aus ihr entnehmen kann, ist die Schrift zwar zum Zeitpunkt dieser eigenstăndigen Tat relevant, aber "... scripture is not decisive precisely because the judgment is imaginative, free, creative" (Kelsey 1975:206).

Eine Theologie ist daher immer ein ästhetisches Gebilde. Obwohl ein theologischer Vorschlag ("proposal") seine auf dieser Basis entwickelte Argumentation nach den Spielregeln der Wissenschaft zu entfalten hat, ${ }^{33}$ ist damit bereits die ästhetische Qualität aller vermittelnden Versuche zwischen antikem Text und heutigem Leser nachgewiesen. Daher erscheint mir die Notwendigkeit der Alternative "Ästhetik" oder "Hermeneutik" nicht legitim. Der letzte Schritt in dieser Gedankenfolge, daß es unter Umständen eher notwendig wäre, beiden gleichermaßen zu ihrem Recht zu verhelfen, erfolgt anhand der zweiten angelsächsischen Reflexion zur "Bible in the modern world".

\subsubsection{Die Bibel als klassisches Modell}

Zwei Jahre früher als Kelsey veröffentlichte James Barr, damals in Oxford, später in Nashville, USA, sein bald berühmt gewordenes Buch The Bible in the Modern World (1973; viele Auflagen folgten), in dem er aus den theologischen Problemen hinsichtlich Status und Verwendung der Bibel seit dem Konsens, der sich nach dem Zweiten Weltkrieg abzeichnete ("the post-war consensus"), und dessen bald folgender Infragestellung Bilanz zieht. Abgesehen von Themen, wie "Inspiration", "Gotteswort" und den

${ }^{33}$ Auch dafür bietet Kelsey im zweiten Teil seiner Ausfuhrungen (1975:122-155) ganz bestimmte Vorschläge. 
kritischen Einwänden radikaler Kreise, wird die Frage nach der Bibel als Literatur eingehend behandelt, und man stößt zu einem Vorschlag für eine Basis als Grundlage zu neuer, konstruktiver Arbeit vor.

\subsubsection{1 "The Bible as Literature"}

Nach Barr ist die Bibel der "foundation myth" des Christentums. Als solcher wird sie als Literatur ästhetisch rezipiert und gewürdigt. Obschon der literarische Mythos auf den Realitäten der menschlichen Existenz basiert, liegt seine Bedeutung nicht in diesem außerliterarischen Bereich (Barr 1973: 52-55). So ist es möglich, daß in der Bibel als Ganzes

“... the parabolic, mythical or literary mode of impact is experienced as the primary one. ... The sense is ... that the story has its main effect anyway before the question of historical events or external realities is answered (Barr 1973:57)".

Diese ästhetische Rezeption der Bibel zeigt sich, neben anderen Funktionen, auch in der liturgischen Verwendung der Heiligen Schrift.

"The Bible provides a series of types or categories of powerful literary character, and these characterize the Christian experience and mark out its distinctive features (Barr 1973:59)".

Es ist klar, daß nach Barr der Sinn nicht nur in der Bibel, aber auch nicht nur in der Erfahrung des christlichen Lesers liegt.

Im Kontext einer der ersten theologischen Darstellungen der Unterscheidung zwischen referentiellem, intentionellem und ästhetischem Gebrauch der Bibel verweist nun Barr darauf, daß manche Aspekte der Bibelverwendung im christlichen Glauben selbst eine enge Verwandtschaft zur religiös neutralen literarischen Wertschätzung zeigen (Barr 1973:60.60 ff.). Vorausgesetzt, daß dieses Studium weder zu einer Vernachlässigung der historisch-kritischen Ergebnisse führt, noch zu einem Deckmantel für eine reaktionäre Verwerfung der historischen Kritik wird und sich auch nicht verabsolutiert, kann es den Zugang zu wichtigen Bedeutungsaspekten der Bibel in der modernen Welt ermöglichen (Barr 1973:73f.).

\subsubsection{Das klassische Modell}

Aber Barr fügt noch einen Schlußstein hinzu. Klar ist, daß ein ästhetischer Zugang zur Bibel tiefen Sinn haben kann. Aber das erübrigt die hermeneutische Perspektive nicht. Das scheint mir die Konsequenz dessen zu sein, daß Barr das Konzept des "klassischen Modells" zum Grundelement seiner konstruktiven Basis macht.

Seine "basic assertion" ist folgende: Der Status der Bibel ist dem Glauben implizit. D.h.: 
"Christian faith is structured upon a certain basic model of the understanding of God. The fundamental model was "first worked out and decisively appropriated" in the Old Testament. That model was reaffirmed, restated and reintegrated in Jesus. Christian faith is faith which relates itself to this classic model. The God in whom Christians believe is the God who was known in the Bible; the Jesus in whom they believe is the Jesus of the New Testament (Barr 1973:115, mit kurzem Zitat von G. D. Kaufman aus 1971)".

Es folgt:

"Faith is Christian because it relates itself to classically-expressed models. ... Christian faith is not whatever a modern Christian may happen to believe, on any grounds at all, but faith related to Jesus and to the God of Israel. The centrality of the Bible is the recognition of the classic sources for the expression of Jesus and of God" (Barr 1973:118).

Das ist gemeint, wenn es heißt, das Christentum sei eine "historische Religion". So ist in Jesus eine "Finalität" erreicht, die aber nicht bedeutet, $\mathrm{da} \beta$ "Offenbarung nun aufhört", sondern daß die Tradition über ihn in ihrer klassischen und rezipierten Form nun zum Rahmen wird, innerhalb dessen zukünftige Ereignisse wahrgenommen und verstanden werden können (Barr 1973:122). Freilich soll man sich davor hüten, das klassische Modell des Gottesverständnisses ${ }^{34}$ zu objektivieren und späteren "supplementary models" aus sekundären Ären der frühen Kirche ${ }^{35}$ Platz einzuräumen, aber die von der Bibel vermittelten Intentionen der klassischen Glaubensgemeinschaft sind für jeden Gebrauch des Textes unumgänglich, wenn christlicher Sinn erfahren werden soll.

Die Frage ist also nicht, ob die Hermeneutik durch die Ästhetik obsolet geworden ist, sondern ob der, der sich ästhetisch mit der Bibel beschäftigt, die ästhetische Erfahrung im Rahmen des christlichen Glaubens wünscht Wenn das der Fall ist, findet die literar-ästhetische Erfahrung stromab im gleichen Fluß statt, in dem die frühe Kirche und Israel ihre Erfahrung mit Gott machten. In diesem Fall muß man per definitionem eine Beziehung zum klassischen Modell herstellen, um sich überhaupt christlich nennen zu können. Die Konsequenz daraus ist wiederum, daß die literarischen Methoden bzw. Zugänge mit historischem Orien-

\footnotetext{
${ }^{34}$ Barr (1973:128): "the classic model of understanding God".

35 Barr (1973:135); z.B. die Modelle der klassischen Christologie und der Trinitätsauffassungen.
} 
tierungspunkt, wie die Formgeschichte ${ }^{36}$ und - vor allem im Lichte des neuesten Stands der Bibelwissenschaften - die Redaktionskritik ${ }^{3}$, unverzichtbar sind. Natürlich muß man nicht mit diesen zutiefst immer noch historisch-kritischen (oder, wenn man das für zu stark gesagt hält, historischen und kritischen) Zugangsweisen arbeiten, um auch im ästhetischen Umgang mit den biblischen Texten Sinn zu erfahren. Aber wenn man Zugang zu Christus haben will, führt der Weg über die dafür geeigneten Methoden. Daher ist es auch nicht angebracht, die Bezeichnung "ästhetisch" für "nichthistorische" oder schlechthin "literarische" Zugänge zur Bibel zu reservieren. Vielmehr scheinen mir diese Überlegungen auf der gleichen Linie zu liegen wie die aneinander anschließenden Horizonte einer ästhetischen und historischen Lektüre ${ }^{38}$. Vielmehr ist die Begegnung mit dem Text in ihrem "existentialen" wie "historischen" Aspekt eine ästhetischc. Das führt aber zu meiner Schlußüberlegung.

\section{SCHLUBÜBERLEGUNGEN AUS DER EIGENEN TRADITION}

Da ich aus einem Teil der angelsächsischen Welt herkomme, der auch durch eine Konjunktur mit der niederländischen Sphäre beeinflußt wurde, sei es mir erlaubt, noch einige aus diesem Zusammenfluß herrührende Bezugspunkte für unser Thema zu erwähnen.

\footnotetext{
${ }^{36}$ Es ist hier nicht der Platz, die Verfeinerung der herkömmlichen Formgeschichte (die mit mündlichen Überlieferungen, den Vorstadien der Texte und deren kleinsten Einheiten arbeitet) zu einer sogenannten "Formengeschichte" (die mit größeren "Makrogattungen" und ihrem schriftlichen Charakter rechnet) zu behandeln, weil der Grundsatz der hier vertretenen These davon nicht berührt wird. Vgl. Körtner (1994:114ff).

37 Aus dieser Perspektive kann Redaktionskritik ruhig auch "Redaktionsgeschichte" heißen und die verschiedenen Phasen dieser Art von historischer Untersuchung eines Textes sind alle jeweils "synchron" (nicht "achron") zu machen.

${ }^{38}$ So weit scheint mir Schunack (1996:29) nicht zu gehen, aber sein Verweis auf die Linie Gadamer-Szondi-Jauß (S. 31) suggeriert doch einen ähnlichen Gedankengang Vgl. Jauß 1984. Zu vergleichen ist auch Szondi (1975:13), der den ästhetischen Charakter des Textes zu einer Prămisse der Auslegung selbst macht und damit eine grundlegende, umfassende ästhetische Würdigung nahelegt. Deist (1991:327-342) ist recht zu geben, wenn er vor einer "premature fusion of horizons" warnt, in der die kulturellen Unterschiede zwischen der Welt, in der der Text produzien wurde, und der Welt, in der er gelesen wird, nicht gebührend bcrücksichtigt werden (S. 335). Seine Forderung in der Formulierung (S. 331f.), wir bräuchten Schutz gegen eine "all-toocasy idealistic and liberal Gadamerian merging of horizons" ist jedoch mindestens wegen ihrer Doppeldeutigkeit fragwürdig.
} 


\subsection{Die sogenannte Ethische Tradition}

Eine theologische Tradition, die den Flügel der südafrikanischen reformierten Theologie, aus dem ich stamme, grundlegend geprägt hat (Loader 1989:412-437; Loader 1996:1-24), kommt aus den Niederlanden und heißt die "ethische Theologie". Nachdem sie ein halbes Jahrhundert lang von der Ideologisierung der Theologie während der Apartheidsära überboten war, kommt sie allmählich wieder zu der ihr gebührenden Anerkennung, und man rechnet wieder damit, daß auch die mageren Dekaden der Bibelwissenschaft vor allem in Pretoria von diesem Einfluß geprägt waren. Die "ethische" Theologie stammt aus den Niederlanden des 19. Jahrhunderts und heißt so wegen ihrer Grundannahme, daß die Wahrheit "ethisch" ist. Die Bezeichnung hat nichts mit dem lateinischen ethica, also nichts mit Moraltheologie zu tun, sondern ist von $\eta \theta 0 \varsigma$ "Charakter", "Wesen" her abzuleiten. Auf einer skeptischen Wirklichkeitsauffassung basierend meint diese Theologie mit dem "ethischen" Charakter der Wahrheit, daß das Wesen der Wahrheit weder objektivier-, noch rationalisier-, noch sonstwie faßbar ist. Aber der Wahrheit kann man sehr wohl begegnen. Gottes Selbstkundgabe bedeutet, daß er sich im Sinne des hebräischen ירע zu erkennen gibt. Also: Wahrheit als Begegnung, nicht als Proposition ${ }^{39}$.

Die ethische Theologie bestand aber ebenso darauf, daß der Mensch verständliche Aussagen über Gott braucht, weil sowohl das menschliche rationale Bedürfnis als auch die soziale Dimension der Kommunikation ${ }^{40}$ das notwendig machen. D.h., vernünftige und verständliche Aussagen ("Propositionen") über Gott sind notwendig als Vermittlung der Zeugnisse von der Wahrheit-als-Begegnung. Sprechen über Gott (oder Sprache über Gott) ist also gnundsätzlich vermittelnd, d.h. hermeneutisch, weil der Sprecher ein Gottesbote ist. Diese soziale Dimension ist die erste von zwei ${ }^{*}$ Beschränkungen des Subjektivismus. Die zweite ist die Bibel, die ja eine Sammlung von Aussagen über menschliche Begegnungen mit Gott bzw. mit der Wahrheit ist und als solche von Haus aus innerhalb einer Gesellschaft und ihrer Nachkommenschaft selbst vermittelnd wirkt (d.h. synchron und diachron). Die Ethiker betonten immer wieder, daß Gottes Selbstkundgabe uns durch die Vermittlung der Schriften Israels und der frühen Kirche progressiv zugänglich gemacht wird (Valeton 1877:14.16). In der Auseinandersetzung mit diesem Traditionsstrom liegt die Identitât der neu

\footnotetext{
${ }^{39}$ Als wäre Emil Brunner ein niederlăndischer Ethiker! Vgl. Brunner 1963 [1938]. Der nunmehr folgende Abriß faßt zusammen, was ich in verschiedenen Schriften herausgearbeitet habe: Loader (1984) (Lit.); (1985:18-32); (1987:47-56).

${ }^{40}$ In dieser Hinsicht unterscheidet sich die Ethische Theologie vom Individualismus des "schweizerischen Schleiermacher" Alexandre Rodolphe Vinet (1797-1847), dessen Einfluß auf die Ethiker ansonsten beträchtlich war, und näherte sich Schleiermachers Betonung der sozialen Dimension der Gemeinde an.
} 
erlebten Offenbarung, Selbstkundgabe Gottes oder Begegnung mit ihm als christliches Erlebnis ${ }^{41}$. In diesem Strom wirkt die Inspiration des Heiligen Geistes, in den Empfängern, d.h. in den Lesern ebenso wie in den ursprünglichen Zeugen. Das letzte Merkmal der ethischen Theologie, auf das ich hinweisen möchte, ist ihr Insistieren auf den geschichtlichen Charakter der Offenbarung Gottes. Sie findet in der Zeit statt, d.h. geschichtlich. Sie fand in einer vergangenen Zeit statt, ${ }^{42}$ wurde bezeugt, fand wieder mit Verweis auf das Modell statt usw., ${ }^{43}$ und ergibt so einen geschichtlichen Traditionsstrom.

\section{2 "Hermeneutische Theologie"}

Es dürfte klar geworden sein, daß hier nicht von einer "allgemeinen" Hermeneutik aus "theologischer" Perspektive geredet wird. Es sollte auch deutlich sein, daß ich den Eröffnungssatz des Vortrags von Oswald Bayer vor der ersten Tagung der Rudolf-Bultmann-Gesellschaft für Hermeneutische Theologie gerne buchstäblich wiederholen möchte: "Christliche Theologie ist hermeneutische Theologie" (Bayer 2000:39) - vielleicht mit anderer Hervorhebung: "Christliche Theologie ist hermeneutische Theologie", um damit dem Fazit meiner Ausführungen Ausdruck zu verleihen. Die Begründung aus der eben skizzierten Tradition wäre allerdings nicht, daß Gott der deus dicens ist. ${ }^{44}$ Daher geht der auch von mir für notwendig gehaltene hermeneutische Charakter der Theologie nicht von einem etwaigen sprachlichen Charakter der Selbstmitteilung Gottes auss. Dic Theologie enthält ihren sprachlichen (propositionellen) Charakter nicht dadurch, daß Gott spricht, ${ }^{46}$ sondern dadurch, daß Menschen sprechen - ist Theo-logie doch nicht Gottes Wort, sonderm ein menschlich reflektives Sprechen über Gott". Gott theologisiert nicht. Ich bekenne mich eher zu dem "Schleiermacher und Bultmann gemeinsame(n) Grundzug", nämlich zu "der Scheu, vom deus dicens direkt und nicht im Spiegel des homo

${ }^{41}$ Offenbar liegt diese Ansicht sehr nahe bei der oben besprochenen Vorstellung Barrs vom "classical model", in dem sich die späteren in einer Beziehung mit den früheren verbunden wissen müssen, um "christlich" zu sein.

${ }^{42} \mathrm{Zu}$ einer bestimmten Zeit in der Geschichte: synchron.

${ }^{43}$ Jeder Punkt in der Zeit ist eine synchron zu verstehende Gelegenheit, und die Reihe, die Tradition oder der Strom der Ubberlieferung ist eine diachronische Abfolge grundsätzlich gleicher Erfahrungen.

${ }^{44}$ Bayer (2000:45): "Weil Gott selbst Hermeneut ist, muß Theologie hermeneutische Theologie sein".

${ }^{45}$ Dagegen meint Bayer (2000:49) eben das.

${ }^{46}$ Erst recht nicht dadurch, daß Gott schreibe (Bayer 2000:46).

${ }^{47}$ Demgegenüber meint O Bayer (2000:45) aus der Etymologie des Wortes "Theologie" herauszuhören, "daß und wie Gott und Wort zueinandergehören". M.E. gehören Gott und des Menschen theologisierendes Wort schon zusammen, aber gerade deshalb, weil

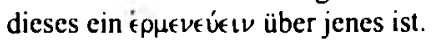


recipiens zu reden" (anders Bayer 2000:41). Wenn die göttliche Selbstoffenbarung, also die Wahrheit, sprachlich wäre, so wäre sie verständlich, also faßbar. Das würde bedeuten, das (um)faßbare Wort umfasse die Wahrheit. Aber finitum non capax infiniti.

Aus diesen Überlegungen heraus erscheint es mir zwar als durchaus berechtigt, den Text zu "transzendieren" (Bayer 2000:51) und den Sinngehalt "im Text und nicht im Urheber des Textes" zu finden, ${ }^{48}$ aber: dann muß der Leser, will er ein christliches Verständnis davon gewinnen, dennoch in einem neuen Zug die Beziehung zur Quelle der christlichen Tradition herstellen, also doch noch in einem "Rückgang hinter den Text auf eine ihm zugrundeliegende Erfahrung" nach dem Sinn als einer Gemeinschaft von ästhetisch in der Rezeption Erlebtem und (ebenso ästhetisch) stromauf in der Begegnung mit Gott Erlebtem fragen. Denn der Heilige Geist hat sich "so sehr an Laute und Buchstaben" gebunden (Bayer 2000:53), wie die christliche Buchtradition zeigt, aber er ist noch immer der Heilige Geist, der für inspirierte Leser und Leserinnen sorgt, wie er für inspirierte Verfasser und - so glaube ich - Verfasserinnen gesorgt hat.

\section{Bibliographie}

Alter, R 1981. The Art of Biblical Narrative. London: Allen \& Unwin.

Alter, R 1981. The Art of Biblical Narrative. New York: Basic Books.

Bal, M, Van Luxemburg, J \& Weststeijn, W G 1983. Inleiding in de Literatuurwetenschap. 3.uit. Muiderberg: Coutinho.

Barr, J 1973. The Bible in the Modern World. The Croall lectures given in New College, Edinburgh in November 1970. London: SCM Press.

- 1979. Review. The Virginia Seminary Journal 31/1, 39.

- 1980. Childs' Introduction to the Old Testament as Scripture. JSOT 16, 12-23.

- 1983. Holy Scripture. Conon, Authority, Criticism. Philadelphia: Westminster.

Barton, J 1984. Reading the Old Testament. Method in Biblical Study. London: Darton, Longman \& Todd.

Bayer, O 2000. Hermeneutische Theologie, in Kortner, U H J $(\mathrm{Hg})$, Glauben und

Verstehen. Perspektiven Hermeneutischer Theologie, 39-55. Neukirchen-Vluyn: Neukirchener Verlag.

Brett, M G 1991. Biblical Criticism in Crisis? The Impact of the Canonical Approach on Old Testament Studies. Cambridge: Cambridge University Press.

Brunner, E [1938] 1963. Wahrheit als Begegnung. 2.Aufl. Zürich: Zwingli.

Bullock, A \& Trombley, S (Ed.), The Fontana Dictionary of Modern Thought. $2^{\text {nd }} \mathrm{ed}$.

London: Fontana Press. (zitiert als FDMT)

Calloud, J 1976. Structural Analysis of Narrative. Philadelphia: Fortress. (SemSup 4.)

Childs, B S 1979. Introduction to the Old Testament as Scripture. Philadelphia: Fortress.

${ }^{48}$ Bayer (2000:52) mit Verweis auf P Ricoeur (1973:194). 
Clines, D J A \& Exum, J C (Ed.) 1993. The New Literary Criticism and the Hebrew Bible. Sheffield: JSOT Press. (JSOT.S 143.)

Culler, J 1982. On Deconstruction. Theory and Criticism after Structuralism. Ithaca: Cornell University Press.

Culley, R C 1974. Structural Analysis: Is it done with Mirrors? Interp. 28, 165-181.

- 1976. Studies in the Structure of Hebrew Narrative. Philadelphia: Fortress. (SemSup 3.)

De Villiers, P G R 1991. The End of Hermeneutics? On New Testament Studies and Postmodernism. Neotestamentica 25/1, 145-156.

Deist, F E 1985. Biblical Hermeneutics, in Deist, F E (Ed.), Study Guide for Biblical Studies. Pretoria.

- 1991. The Bible as Literature: Whose Literature? OTE 7/3, 327-342.

Detweiler, R (Ed.) 1985. Reader Response Approaches to Biblical and Secular Texts. Decatur: Scholars Press. (Semeia 31.)

Dohmen, C \& Stemberger, G 1996. Hermeneutik der jüdischen Bibel und des Alten Testaments. Stuttgart: Kohlhammer. (KStTh 1/2.)

Ebeling, G 1969. Hermeneutische Theologie? In Ebeling, G (Hg.), Wort und Glaube, Bd. 2, Beiträge zur Fundamentaltheologie und zur Lehre von Gott, 99-120. Tübingen: Mohr.

Exum, J C 1996. Plotted, Shot, and Painted: Cultural Representations of Biblical Women. Sheffield: Sheffield Academic Press. (JSOT.S 215; Gender, Culture, Theory 3.)

Forget, P (Hg.) 1984. Text und Interpretation. Deutsch-französische Debatte. München: Fink. (UTB 1257.)

Gadamer, H-G [1960] 1986. Wahrheit und Methode. Grundzüge einer Philosophischen Hermeneutik. 5.Aufl. Tübingen: Mohr.

Groenewald, E P 1969. Krisis in die Interpretasie van die Heilige Skrif. NGTT 10, 2-12.

Grondin, J 1991. Einführung in die philosophische Hermeneutik. Darmstadt: Wissenschaftliche Buchgesellschaft. (Einführung; Die Philosophie.)

Hawkes, T 1977. Structuralism and Semiotics. London: Methuen. (New accents.)

Hunter, J H 1991. Deconstruction and Old Testament. An Evaluation of "Context" with Reference to 1 Samuel 9:2. OTE 4/3, 362-372.

Ingarden, R 1972. Das literarische Kunstwerk. 4.Aufl. Tübingen: Niemeyer.

Iser, W 1976. Der Akt des Lesens. Theorie ästhetischer Wirkung. München: Fink. (UTB 636.)

- 1984. Der Akt des Lesens. Theorie ästhetischer Wirkung. 2.Aufl. München: Fink. (UTB 636.)

Jackson, J \& Kessler, M (Ed.) 1974. Rhetorical Criticism. Essays in Honor of James Muilenburg. Pittsburgh: Pickwick. (PThMS 1.)

Jauß, H R 1984. Ästhetische Erfahrung und literarische Hermeneutik. 4.Aufl. Frankfurt am Main: Suhrkamp.

Jobling, D \& Moore, S D (Ed.) 1992. Poststructuralism as Exegesis. Atlanta: Scholars Press. (Semeia 54.)

Kelsey, D H 1975. The Uses of Scripture in recent Theology. Philadelphia: Fortress. 
Körtner, U H J 1999. Perspektiven Hermeneutischer Theologie im Anschluß an Rudolf Bultmann. BThZ 16/2, 258-266.

Körtner, U H J 1994. Der inspirierte Leser. Zentrale Aspekte biblischer Hermeneutik.

Göttingen: Vandenhoeck \& Ruprecht. (Sammlung Vandenhoeck.)

Lategan, B C 1970. Voorlopers van die ,Nuwe Hermeneutiek'. NGTT 11, 22-28.

- 1989. Coming to Grips with the Reader in Biblical Literature, in McKnight, E V (ed.),

Reader Perspectives on the New Testament, 3-. Atlanta: Scholars Press. (Semeia 48.)

Loader, J A 1979. The Use of the Bible in Conventional South African Theology, in Vorster, W S (Ed.), Scripture and the Use of Scripture, 1-25. Pretoria: Unisa.

- 1984. Die etiese Ou-Testamentici in Nederland tussen 1870 en 1914. Dissertation.

Pretoria.

- 1985. The Concept of Revelation in Dutch Ethical Theology. OTE 3, 18-32.

- 1987. Tertium datur - oor die etiese Waarheidsbegrip. HTS 43/1, 47-56. (in diesem

Band: Tertium datur. Zum Wahrheitsbegriff der "Ethischen Theologie".)

- 1989. Ontstaan en eerste periode van die Hervormde teologiese opleiding aan die Universiteit van Pretoria. HTS 45/2, 412-437.

- 1996. 'n Hervormde tradisie as heelmiddel. HTS 52/4, 1-24.

Louw, J P 1973. Discourse Analysis and the Greek New Testament. Bible Translator 24, 101-118.

Mack, B 1990. Rhetoric and the New Testament. Minneapolis: Fortress. (Guides to biblical scholarship - New Testament series.)

Moore, S D 1989. Literary Criticism and the Gospels. The Theoretical Challenge. New Haven: Yale University Press.

Muilenburg, J 1969. Form Criticism and Beyond. JBL 88,1-18.

Nethöfel, W 1992. Theologische Hermeneutik. Vom Mythos zu den Medien. Neukirchen-Vluyn: Neukirchener Verlag. (NBST.)

Oeming, M 1998. Biblische Hermeneutik. Eine Einführung. Darmstadt: Wissenschaftliche Buchgesellschaft. (Einführung; Die Theologie.)

Patte, D 1976. What is Structural Exegesis? Philadelphia: Fortress. (New Testament series.)

Poland, L M 1985. Literary Criticism and Biblical Hermeneutics. A Critique of Formalist Approaches. Chico: Scholars.

Polzin, R M 1977. Biblical Structuralism. Method and Subjectivity in the Study of Ancient Texts. Philadelphia: Fortress. (SemSup 5.)

Powell, M A 1990. What is Narrative Criticism? Minneapolis: Fortress. (Guides to biblical scholarship - New Testament series.)

Prinsloo, W S 1988. The cohesion of Haggai 1:4-11, in Augustin, M \& Schunck, K-D (Hg.), "Wünschet Jerusalem Frieden", Collected communications to the XIIth congress of the International Organization for the Study of the Old Testament 12, Jerusalem 1986, 337-343. Frankfurt am Main, u.a.: Lang. (BEAT 13.)

- 1992. The Unity of the Book of Joel. ZAW 104, 66-81.

Ransom, J C 1941. The New Criticism. Norfolk: New Directions.

Rendtorff, R 1992. Das Alte Testament. Eine Einführung. 4.Aufl. Neukirchen-Vluyn:

Neukirchener Verlag. 
Ricoeur, P 1973. Hermeneutik und Strukturalismus. München: Kösel. (Paul Ricoeur: Der Konflikt der Interpretationen 1.)

Roberts, J H 1973. Rondom die Hermeneutiek. ThEv(SA) 6, 138-154.

Sanders, J A 1984. Canon and Community. A Guide to Canonical Criticism. Philadelphia: Fortress. (Guides to biblical scholarship - Old Testament series.)

Scholder, K 1971. Geleitwort. VF 16/1.

Schunack, G 1996. Neuere literaturkritische Interpretationsverfahren in der angloamerikanischen Exegese. $V(u) F 41,28-55$.

Seymore-Smith, M 1988. S.v. "New Criticism", FDMT, 579.

Staiger, E 1955. Die Kunst der Interpretation. Studien zur deutschen Literaturgeschichte. Zürich: Atlantis.

Suleiman, S R \& Crosman, I (Ed.) 1980. The Reader in the Text. Essays on Audience and Interpretation. Princeton: Princeton University Press.

Sundberg, A C 1964. The Old Testament of the Early Church. Cambridge: Harvard University Press. (HThS 20.)

Szondi, P 1975. Einfuhrung in die literarische Hermeneutik. Frankfurt: Suhrkamp. (stw 124.)

Tillich, P 1963. Systematic Theology. Bd. 1, Reason and revelation, Being and God. Chicago: University of Chicago Press.

- 1963. Systematic Theology. Bd. 3, Life and the spirit. History and the kingdom of God. Chicago: University of Chicago Press.

Valeton, J J P (Jr.) 1877. De Israelitische Letterkunde als onderdeel der Christelike Theologie. Groningen: Wolters.

Warfield, B 1948. The Inspiration and Authority of the Bible. Phillipsburg: Presbyterian and Reformed Publishing Company.

Warner, M (Ed.) 1990. The Bible as Rhetoric. Studies in Biblical Persuasion and Credibility. London u.a.: Routledge. (Warwick studies in philosophy and literature.)

Wellek, R \& Warren, A 1963. Theory of Literature. $3^{\text {rd }}$ ed. Harmondsworth: Penguin Books. (Peregrine books 28.)

Wright, G E 1952. God who Acts. Biblical theology as recital. London: SCM Press. (SBT 8.)

Wuellner, W 1987. Where is Rhetorical Criticism taking us? $C B Q 49,448-463$.

Zenger, E (Hg.) 1996. Einleitung zum Alten Testament. 2.Aufl. Stuttgart: Kohlhammer. (KStTh 1/1.) 